Do Changes in Perioperative and Postoperative Treatment Protocol Influence the Frequency of Pulmonary Complications? A Retrospective Analysis of Four Different Bariatric Groups

\author{
Javanainen, Mervi Hannele
}

2017-01

Javanainen , M H , Scheinin , T , Mustonen , H \& Leivonen , M 2017 , ' Do Changes in Perioperative and Postoperative Treatment Protocol Influence the Frequency of Pulmonary Complications? A Retrospective Analysis of Four Different Bariatric Groups ' , Obesity Surgery , vol. 27 , no. 1 , pp. 64-69 . https://doi.org/10.1007/s11695-016-2236-7

http://hdl.handle.net/10138/237257

https://doi.org/10.1007/s11695-016-2236-7

publishedVersion

Downloaded from Helda, University of Helsinki institutional repository.

This is an electronic reprint of the original article.

This reprint may differ from the original in pagination and typographic detail.

Please cite the original version. 


\title{
Do Changes in Perioperative and Postoperative Treatment Protocol Influence the Frequency of Pulmonary Complications? A Retrospective Analysis of Four Different Bariatric Groups
}

\author{
Mervi Hannele Javanainen ${ }^{1} \cdot$ Tom Scheinin $^{1} \cdot$ Harri Mustonen $^{1} \cdot$ Marja Leivonen $^{1}$
}

Published online: 24 May 2016

(C) Springer Science+Business Media New York 2016

\begin{abstract}
The current understanding of prophylaxis of pulmonary complications in bariatric surgery is weak. Purpose: The aim of this study was to observe how changes in perioperative and postoperative treatments affect the incidence of pulmonary complications in bariatric patients. Materials: This is a retrospective clinical study of 400 consecutive bariatric patients. The patients, who either underwent a sleeve gastrectomy or a Roux-en-Y gastric bypass, were divided consecutively into four subgroups with different approaches to perioperative treatment. Methods: The first group (patients 0-100) was recovered in the intensive care unit with minimal mobilization (ICU). They had a urinary catheter and a drain. The second group (patients 101-200) was similar to the first group, but the patients used a continuous positive airway pressure (CPAP) device intermittently (ICU-CPAP). The third group (patients 201-300) was recovered on a normal ward without a urinary catheter or a drain and used a CPAP device (ward-slow). The fourth group (patients 301-400) walked to the operating theater and was mobilized in the recovery room during the first $2 \mathrm{~h}$ after the operation (ward-fast). CPAP was also used. Primary endpoints were pulmonary complications, pneumonia, and infection, non-ultra descriptus (NUD). Results: The number of pulmonary complications among the groups was significantly different. A long operation time increased the risk for infection $(p<0.00195 \% \mathrm{CI}$ from 2.02 to $6.59 \%$ ). Conclusions: Operation time increases the risk for pulmonary complications. Changes in perioperative care toward the ERAS protocol may have a positive effect on the number of pulmonary complications.
\end{abstract}

Mervi Hannele Javanainen mervi.javanainen@hus.fi

1 Peijas Hospital, HUS, Lohja, Finland
Keywords Bariatric surgery $\cdot$ Pulmonary complications · ERAS

\section{Introduction}

Most of the of world's population live in countries where being overweight kills more people than being underweight do [1]. Surgical treatment of morbid obesity has been proven to be safe [2] and has been gaining in popularity and acceptance over the past years. Like any other surgical procedure, the benefits of the operation are influenced by its morbidity and mortality [3].

Obesity affects pulmonary function and can significantly alter cardiopulmonary physiology [4]. These alterations are a combination of mechanical- and inflammatory-mediated changes that can result in pulmonary dysfunction [4]. Obstructive sleep apnea (OSA) is more common among obese patients. With bariatric surgery, it is possible to improve OSA in the vast majority of patients [5]. Obesity is also associated with a $50 \%$ increase in the incidence of asthma compared to normal-weight patients [6].

Gupta demonstrated in his large cohort study that postoperative pneumonia and respiratory failure account for one fifth of the total morbidity of $5 \%$ in bariatric surgery [3]. Congestive heart failure and stroke preoperatively were the greatest risk factors for pneumonia, while other risk factors were age, COPD, smoking, and diabetes [3]. In a review of 11,023 patients, the incidence of pneumonia varied between 0 and $2.2 \%$ between different bariatric operation types, the overall incidence being $0.6 \%$ [7].

Pulmonary comorbidities can often be underdiagnosed in bariatric patients [4]. It is recommended by the Interdisciplinary European Guidelines on metabolic and bariatric surgery that bariatric surgery patients should undergo assessments for sleep apnea 
syndrome and pulmonary function prior to surgery [8]. A multidisciplinary approach with an anesthesiologist and pulmonary specialist while planning the operation can be beneficial [4].

For postoperative pulmonary management, American Guidelines for the Clinical Practice for the Perioperative Nutritional, Metabolic, and Nonsurgical Support of the Bariatric Surgery recommend aggressive pulmonary toilet, spirometry, oxygen supplementation, and the use of continuous positive airway pressure (CPAP) when clinically indicated [9]. CPAP is a breathing mode in which continuous positive pressure is maintained during both inspiration and expiration in spontaneously breathing patients. Postoperative use of CPAP is often recommended [10]. In a recent meta-analysis of randomized controlled trials where CPAP was compared to standard care preventing postoperative morbidity after major abdominal surgery, no clear evidence between the control group and the CPAP group was found [11]. In bariatric surgery, similar results have been presented $[10,12]$.

Enhanced recovery after surgery (ERAS) was initially invented by Henrik Kehlet in the 1990s. The content of the different ERAS protocols vary, but principles usually include the use of minimally invasive surgical techniques and aggressive postoperative rehabilitation, including early oral nutrition and early removal of tubes and urine catheters [13]. There is ample evidence on ERAS programs in other types of surgery, for example, colorectal surgery $[14,15]$, but results in bariatric surgery are still unclear [16].

The current understanding of the prophylaxis of pulmonary complications after obesity surgery is weak, and to our knowledge, there are only few publications on this topic [17]. Our study setting was a clinical study, and the aim was to observe how changes in perioperative and postoperative treatments affect the incidence of pulmonary complications in bariatric patients.

\section{Methods}

This is a retrospective, non-randomized, clinical, single center study of the first consecutive 400 patients who underwent bariatric surgery between the years 2008 and 2011 in Peijas Hospital, the Bariatric Surgery Unit of Helsinki University Central Hospital. The study included both primary and revisional bariatric surgery patients.

Patient selection was similar to the Interdisciplinary European Guidelines on Metabolic and Bariatric Surgery recommendations [8]. Patients had a body mass index over $40 \mathrm{~kg} / \mathrm{m}^{2}$ or over $35 \mathrm{~kg} / \mathrm{m}^{2}$ with comorbidities, like diabetes or hypertension. Patients also had failed to lose weight or to maintain long-term weight loss, despite appropriate nonsurgical medical care. Prior to surgery, patients underwent extensive medical, nutritional, and psychological screening. All patients met the surgeon 3 to 6 weeks after the operation and were thereafter followed by an endocrinologist and a nutritionist.

At the beginning, when bariatric operations were started de novo in Peijas Hospital, there were no guidelines available on how to prevent pulmonary complications in bariatric patients. During our learning curve, we noticed a large number of pulmonary complications or obscure infections on the ward in our first 100 patients (the ICU group). Inspired by this observational data, we started to systematically develop our postoperative treatment strategy for the next hundred patients (ICU-CPAP group). After the first 200 patients, we still were not happy with the pulmonary and infectious complication rate and made again changes to the protocol for the next hundred patients (ward-slow group). After this group, we noticed a positive change and decided to modify the protocol once more (ward-fast group). Finally, we had four different subgroups, each including 100 patients, with different perioperative protocols. We opted for an even number for the group size so groups could be easily compared. For ethical reasons, the group size had to be relatively small because of the large number of pulmonary complications observed in the first groups.

The ICU group (patients $0-100$ ) recovered in the intensive care unit (ICU) with minimal mobilization according to the treatment principles of the ICU, which are focused on seriously ill patients. They had a urinary catheter, a drain, and an arterial blood pressure monitoring. Patients also did bottle blow exercises 10 times per day, 10 times per exercise. The ICU-CPAP group (patients 101-200) also recovered in the ICU with a urinary catheter, a drain, and an arterial needle and they did their bottle blow exercises, but they also used a CPAP device intermittently four times per day, $1 \mathrm{~h}$ at a time. The ward-slow group (patients 201-300) recovered on a normal ward with normal daily rehabilitative routines and without a urinary catheter, a drain, or an arterial needle. They did their bottle blow exercises as the other groups, and their CPAP therapy was started in the recovery room immediately after the operation. On the ward, they had CPAP four times per day, continuing $1 \mathrm{~h}$ at a time. The ward-fast group (patients 301400) walked to the operating theater and was mobilized in the recovery room during the first $2 \mathrm{~h}$ after the operation. Bottle blow exercises and CPAP were used as in the previous groups. In all groups, pain medication was given mainly orally avoiding opioids. All patients also received pharmacological thromboembolic prophylaxis of enoxaparin. Antibiotic prophylaxis was given during the hospital stay with standard doses.

Total intravenous anesthesia (TIVA) was used with all the patients, without a few isolated patients. Esketamine has been used with all the patients before the end of the anesthesia.

As primary endpoints, we used pneumonia and nonspecific infection NUD. Pneumonia was diagnosed with elevated infection parameters and radiologic findings in a chest 
X-ray or computed tomography $(\mathrm{CT})$ scan. Infection NUD was considered to be a postoperative fever with elevated infection parameters (leukocyte count and c-reactive protein) leading to a longer hospital stay or antimicrobial medication to home. In infected NUD, no clear imaging finding was obtained, but atelectasis of lungs was frequently seen. As secondary endpoints, we monitored operation time and the number of reoperations and duration of the hospital stay. Data was collected prospectively. The study setting had no ethical controversies.

Statistical analysis was carried out using SPSS version 21 (IMB Corp., New York, NY). Continuous data were tested for normality using the Shapiro-Wilk test. Results were given as mean/standard deviation (SD) and number/percentage of patients. Differences between groups in continuous variables were tested with the Kruskal-Wallis test or Mann-Whitney U test and differences in binominal or categorical variables with Fisher's exact test or Fisher-Freeman-Halton test. Logistic regression analysis was used and results given as an odds ratio and $95 \%$ confidence intervals (CIs). Group, sex, COPD, asthma, OSA, and operation time were included in the model.

\section{Results}

Of the total 400 patients, $34 \%$ were men. Mean age was 47.7 \pm 9.1 years, ranging from 23 to 67 years, and the mean preoperative body mass index was $48.6 \pm 6.9 \mathrm{~kg} / \mathrm{m}^{2}$. In our study, $53.5 \%$ of the patients had diabetes, $30.8 \%$ sleep apnea, $16.8 \%$ asthma, $1.25 \%$ COPD, and $73.2 \%$ hypertension The four groups were similar with each other regarding age, preoperative comorbidities, and preoperative weight (Table 1).
In the ward-slow and ward-fast groups, the number of different operation types varied compared to the ICU and ICUCPAP groups. This was because at the time of these groups, our hospital took part in a multicenter study in which the type of operation was randomized between gastric bypass and sleeve procedure [18]. There were $17(4.3 \%)$ revision operations. Gastric banding was converted to a sleeve or gastric bypass in 13 of these, a band was removed in 3 cases, and a sleeve was turned into a gastric bypass in 1 case.

The incidence of pneumonia and infected NUD was compared among groups. The total number of pneumonia cases was $48(12 \%)$ and infected NUD $30(7.5 \%)$ (Table 2).In the logistic regression model (Table 3), the risk for pneumonia and infected NUD was significantly different between the ICU group and the ward-fast group. In the logistic regression model, age, asthma, COPD, or OSA did not reach significance (Table 3). A long operation time increased the risk for infection (Table 4). The difference in operation time between the patients with or without pulmonary complications was significant (Table 4).

In order to study the learning curve, we compared the first 50 and second 50 patients in the ICU group. We found a difference between the operation times $128 / 100 \mathrm{~min}$, respectively $(p=0.00)$, but there was no difference in the number of pulmonary complications $12 / 8$, respectively $(p=0.45)$.

There were three reoperations in the ICU group: one gastrojejunal (GJ) anastomotic leakage and two bleedings from the gastric remnant. There were also three reoperations in the ICU-CPAP group: two enteroanastomotic (EA) stenosis and one EA strangulation. In the ward-slow group, there was one reoperation for GJ anastomotic leakage, and in the wardfast group, there were five reoperations: one intraabdominal

Table 1 Patient data

\begin{tabular}{|c|c|c|c|c|c|c|}
\hline Group & & $\begin{array}{l}\text { ICU group } \\
(n=100)\end{array}$ & $\begin{array}{l}\text { ICU-CPAP group } \\
(n=100)\end{array}$ & $\begin{array}{l}\text { Ward-slow group } \\
(n=100)\end{array}$ & $\begin{array}{l}\text { Ward-fast group } \\
(n=100)\end{array}$ & $p$ value \\
\hline Age, mean $( \pm \mathrm{SD})$ & & $47.90(8.8)$ & $47.80(8.8)$ & $47.60(9.7)$ & $47.6(8.8)$ & 0.993 \\
\hline Male $\%$ & & 40 & 32 & 32 & 29 & 0.390 \\
\hline Preoperative BMI $\left(\mathrm{kg} / \mathrm{m}^{2}\right)( \pm \mathrm{SD})$ & & $48.9(6.7)$ & $48.9(6.8)$ & $48.7(6.5)$ & $48.1(7.7)$ & 0.663 \\
\hline \multicolumn{7}{|l|}{ Comorbidities (\%) } \\
\hline & Diabetes & 59 & 56 & 50 & 49 & 0.429 \\
\hline & Sleep apnea & 36 & 22 & 33 & 32 & 0.150 \\
\hline & Asthma & 13.0 & 14.0 & 17.0 & 23.0 & 0.238 \\
\hline & COPD & 0.0 & 0.0 & 1.0 & 5.0 & 0.017 \\
\hline & Hypertension & 68.0 & 74.0 & 74.0 & 76.0 & 0.609 \\
\hline
\end{tabular}

The ICU group (patients 0-100) recovered in the intensive care unit (ICU) with minimal mobilization. They had a urinary catheter and a drain. They also made bottle blow exercises 10 times per day, 10 times per exercise; the ICU-CPAP group (patients 101-200) recovered in the ICU with a urinary catheter, a drain, and they made bottle blow exercises, but they also used a CPAP device intermittently four times per day, $1 \mathrm{~h}$ at a time; the ward-slow group (patients 201-300) recovered on a normal ward with normal daily rehabilitative routines and without a urinary catheter or a drain. They did their bottle blow exercises as the other groups, and their CPAP therapy was started in the recovery room immediately after the operation; the ward-fast group (patients 301-400) walked to the operating theater and was mobilized in the recovery room during the first $2 \mathrm{~h}$ after the operation. Bottle blow exercises were made and CPAP were used as in the previous groups 
Table 2 Operation time, in-hospital time, pneumonia, and infection NUD

\begin{tabular}{|c|c|c|c|c|c|}
\hline Group & $\operatorname{ICU}(n=100)$ & $\mathrm{ICU}+\mathrm{PAP}(n=100)$ & Ward-slow $(n=100)$ & Ward-fast $(n=100)$ & $p$ value \\
\hline Pneumonia (\%) & 20 & 12 & 10 & 6 & 0.020 \\
\hline Infection NUD (\%) & 11 & 8 & 6 & 5 & 0.387 \\
\hline Operation time/min $( \pm \mathrm{SD})$ & $114.3(37)$ & $95.3(28.1)$ & $81.4(29.5)$ & $82.0(25.5)$ & $<0.001$ \\
\hline Hospital stay/day $( \pm \mathrm{SD})$ & $6.13(4.9)$ & $5.90(4.1)$ & $5.06(1.4)$ & $4.60(2.4)$ & $<0.001$ \\
\hline Number of reoperations & 3 & 3 & 1 & 5 & 0.474 \\
\hline
\end{tabular}

The ICU group (patients 0-100) recovered in the intensive care unit (ICU) with minimal mobilization. They had a urinary catheter and a drain. They also made bottle blow exercises 10 times per day, 10 times per exercise; the ICU-CPAP group (patients 101-200) recovered in the ICU with a urinary catheter, a drain, and they made bottle blow exercises, but they also used a CPAP device intermittently four times per day, $1 \mathrm{~h}$ at a time; the ward-slow group (patients 201-300) recovered on a normal ward with normal daily rehabilitative routines and without a urinary catheter or a drain. They did their bottle blow exercises as the other groups, and their CPAP therapy was started in the recovery room immediately after the operation; the ward-fast group (patients 301-400) walked to the operating theater and was mobilized in the recovery room during the first $2 \mathrm{~h}$ after the operation. Bottle blow exercises were made and CPAP were used as in the previous groups

bleeding, one EA leakage, one leakage in the GJ anastomosis, one anastomotic bleeding, and one suspected strangulation (Table 2). There were no statistical difference in the amount of reoperations between the groups $(p=0.47)$

The mean hospital stay was 5 days varying from 2 to 39 days, and there was a significant difference in the hospital stay between the groups (Table 2). Also, patients without infection complications had a shorter in-hospital time compared to those who had pneumonia/infection NUD (Table 4).

\section{Discussion}

Postoperative pulmonary complications are common and are associated with increased mortality and morbidity [19]. Respiratory complications have also been proven to increase the length of the hospital stay and increase the cost of hospital treatment [20].

In a study of 11,023 bariatric surgery patients, the incidence for pneumonia varied from 0 to $2.2 \%$ [7]. In our study, the overall incidence of pneumonia was $12 \%$. Reasons for this high incidence can be hypothesized. We aggressively monitored postoperative problems and imaging studies were performed with a low threshold. The comparison among studies can also be unreliable because the definition for pneumonia varies greatly in the literature.

ERAS is an incorporated multiple evidence-based perioperative intervention to accelerate and standardize patient recovery without increasing the incidence of postoperative complications [21]. Its principles include aggressive postoperative rehabilitation, including early removal of tubes and urine catheters and early oral nutrition [13].
Table 3 Logistic regression analysis for pulmonary complications regarding group, gender, asthma, COPD, OSA, and operation time

\begin{tabular}{llllll}
\hline Variable & & ODDS & $95 \%$ CI lower & $95 \%$ CI higher & $p$ value \\
\hline Group & ICU & 1.00 & & & \\
& ICU + CPAP & 0.65 & 0.33 & 1.28 & 0.212 \\
& Ward-slow & 0.57 & 0.28 & 1.18 & 0.132 \\
& Ward-fast & 0.32 & 0.14 & 0.74 & 0.008 \\
Sex & Female vs male & 1.70 & 0.90 & 3.22 & 0.100 \\
Asthma & Yes vs. no & 1.93 & 1.00 & 3.73 & 0.051 \\
COPD & Yes vs. no & 2.95 & 0.43 & 20.24 & 0.272 \\
OSA & Yes vs. no & 0.92 & 0.49 & 1.76 & 0.810 \\
Operation time & $>88$ vs. $\leq 88$ min & 3.65 & 2.02 & 6.59 & $<0.001$ \\
\hline
\end{tabular}

The ICU group (patients 0-100) recovered in the intensive care unit (ICU) with minimal mobilization. They had a urinary catheter and a drain. They also made bottle blow exercises 10 times per day, 10 times per exercise; the ICU-CPAP group (patients 101-200) recovered in the ICU with a urinary catheter, a drain, and they made bottle blow exercises, but they also used a CPAP device intermittently four times per day, $1 \mathrm{~h}$ at a time; the ward-slow group (patients 201-300) recovered on a normal ward with normal daily rehabilitative routines and without a urinary catheter or a drain. They did their bottle blow exercises as the other groups, and their CPAP therapy was started in the recovery room immediately after the operation; the ward-fast group (patients 301-400) walked to the operating theater and was mobilized in the recovery room during the first $2 \mathrm{~h}$ after the operation. Bottle blow exercises were made and CPAP were used as in the previous groups

$C O P D$ chronic obstructive pulmonary disease; OSA obstructive sleep apnea 
Table 4 Operation time and hospital stay

\begin{tabular}{llll}
\hline & No infection & Pneumonia or infection NUD & $p$ value \\
\hline Operation time $(\min )( \pm \mathrm{SD})$ & $88(30)$ & $114(39)$ & $<0.001$ \\
Hospital time (day) $( \pm \mathrm{SD})$ & $5.2(3.5)$ & $6.3(3.4)$ & $<0.001$ \\
\hline
\end{tabular}

In a recently published, randomized, controlled trial, 116 patients were divided into three groups after laparoscopic sleeve gastrectomy: 78 patients to the ERAS group, 40 patients to a control group, and 38 patients in a historical group. The median hospital stay in the ERAS group was significantly shorter than in the other groups, with no difference in postoperative complication rates. In the study, perioperative management in the ERAS group included early oral intake, mobilization after $2 \mathrm{~h}$ returning to a ward, standardized analgesia, and antithrombotic prophylaxis [22]. The current literature has yet to define which aspects of the ERAS method will lead to better results in bariatric surgery.

Prophylactic drainage has been used in the setting of Rouxen-Y gastric bypass (RYGBP) to detect early anastomotic leaks [23]. There is only slight evidence of its benefit in bariatric surgery [24], and prophylactic drainage might be unnecessary in this type of surgery, as shown in other types of abdominal surgery [25]. In our study, the use of urinary catheters and drains was halted after 200 patients because of the lack of a clear benefit. It can be hypothesized that catheters and drains can work as infection ports as well as increase the pain experienced by patients, slowing down the mobilization.

In our study, a modified ERAS protocol was used in the two last groups with no urinary catheters or drains and with aggressive mobilization especially in the last patient group. Our results showed that the incidence of pneumonia and non-specific infections decreased compared to each previous group, suggesting that changes in perioperative management can result in fewer complications.

There is a positive correlation between the length of anesthesia and postoperative pulmonary complications [3]. In a large meta-study of 32,889 patients, the mean anesthesia time for patients was 208 min with pulmonary complications and 160 min without [3]. In our study, we saw a similar trend. The operating time was longer in patients with pulmonary complications although the operation time in our patients was generally shorter than that in the meta-analysis.

A learning curve of 50-100 patients before gaining proficiency in bariatric Roux-en-Y gastric bypass has been suggested [26]. In our study, the operation time significantly decreased after the first 50 patients, indicating the learning curve $(p=0.00)$. In the first 50 patients and second 50 patients, the operation time was $128 / 100 \mathrm{~min}$, respectively, $(p=000)$. The operation time decreased compared to each previous group, and operation time was found to be a correlation factor in the logistic regression model for pulmonary complications. Therefore, the conclusion that changes in perioperative management could affect the number of pulmonary complications must be taken with caution. However, no difference was seen in the number of pneumonia cases in the first and second 50 patients. The figures were $38 / 42$, respectively, $(p=0.45)$, suggesting that the operation time was not the only factor affecting the number of complications.

Obese asthma patients have worse asthma control than do normal-weight patients [27], and this might make them more vulnerable to postoperative pulmonary complications [28]. In our study, no significant correlation between the incidence of pneumonia and asthma was found. However, the group of patients with asthma was relatively small.

Bariatric surgery can lead to full or partial remission of OSA syndrome [29] and improve asthma severity with weight loss [30]. Although patients with pulmonary comorbidities are prone to complications, they should not be excluded from bariatric surgery as they can benefit greatly from the operation. Instead, with these patients, optimizing the perioperative care to avoid complications is extremely important. A correlation has been found between spirometry results and pulmonary and other complications after bariatric surgery [28]. In the future, one model to improve the outcome of bariatric surgery may be pulmonary function testing preoperatively.

The strength of this study was its dynamic study setting, which allowed us to follow current trends in perioperative care. The present study also had several limitations: first, its observational nature and the lack of randomization of the patients; second, we lacked the preoperative data of patients' smoking habits, which have been linked to increased pulmonary complications [30]; and third, the definition for the infection NUD, fever, and elevated infection parameters. Fever postoperatively can also result from other inflammatory or reactive processes, for example, delirium or pulmonary embolism [31]. However, in our study, these were excluded, and the most probable causes for fever remain respiratory malfunction and atelectasis.

In conclusion, operation time increases the risk for pulmonary complications. Changes in perioperative care toward ERAS principles may have a positive effect on the frequency of pulmonary complications.

\section{Compliance with Ethical Standards}

Conflict of Interest The authors declare that they have no conflicts of interest.

Informed Consent Informed consent was obtained from all individual participants included in the study. 


\section{References}

1. WHO:Obesity and overweight: Fact sheet N311 Updated January 2015

2. Dumon KR, Murayama KM. Bariatric surgery outcomes. Surg Clin North Am 2011; 91:1313,38, x.

3. Gupta PK, Gupta H, Kaushik M, et al. Predictors of pulmonary complications after bariatric surgery. Surg Obes Relat Dis. 2012;8:574-81.

4. Davis G, Patel JA, Gagne DJ. Pulmonary considerations in obesity and the bariatric surgical patient. Med Clin North Am 2007 91:433, 42, xi.

5. Sarkhosh K, Switzer NJ, El-Hadi M, et al. The impact of bariatric surgery on obstructive sleep apnea: a systematic review. Obes Surg. 2013;23:414-23.

6. Beuther DA, Sutherland ER. Overweight, obesity, and incident asthma: a meta-analysis of prospective epidemiologic studies. Am J Respir Crit Care Med. 2007;175:661-6.

7. Gupta PK, Franck C, Miller WJ, et al. Development and validation of a bariatric surgery morbidity risk calculator using the prospective, multicenter NSQIP dataset. J Am Coll Surg. 2011;212:301-9.

8. Fried M, Yumuk V, Oppert JM, et al. Interdisciplinary European Guidelines on metabolic and bariatric surgery. Obes Surg. 2014;24: $42-55$.

9. Mechanick JI, Youdim A, Jones DB, et al. Clinical practice guidelines for the perioperative nutritional, metabolic, and nonsurgical support of the bariatric surgery patient -2013 update: cosponsored by American Association of Clinical Endocrinologists, The Obesity Society, and American Society for Metabolic \& Bariatric Surgery. Obesity (Silver Spring). 2013;21 Suppl 1:S1-27.

10. Jensen C, Tejirian T, Lewis C, et al. Postoperative CPAP and BiPAP use can be safely omitted after laparoscopic Roux-en-Y gastric bypass. Surg Obes Relat Dis. 2008;4:512-4.

11. Ireland CJ, Chapman TM, Mathew SF, et al. Continuous positive airway pressure (CPAP) during the postoperative period for prevention of postoperative morbidity and mortality following major abdominal surgery. Cochrane Database Syst Rev. 2014;8:CD008930.

12. Huerta S, DeShields S, Shpiner R, et al. Safety and efficacy of postoperative continuous positive airway pressure to prevent pulmonary complications after Roux-en-Y gastric bypass. J Gastrointest Surg. 2002;6:354-8.

13. Wilmore DW, Kehlet H. Management of patients in fast track surgery. BMJ. 2001;322:473-6.

14. Gouvas N, Tan E, Windsor A, et al. Fast-track vs standard care in colorectal surgery: a meta-analysis update. Int J Colorectal Dis. 2009;24:1119-31.

15. Eskicioglu C, Forbes SS, Aarts MA, et al. Enhanced recovery after surgery (ERAS) programs for patients having colorectal surgery: a meta-analysis of randomized trials. J Gastrointest Surg. 2009;13: 2321-9.

16. Lemanu DP, Srinivasa S, Singh PP, et al. Optimizing perioperative care in bariatric surgery patients. Obes Surg. 2012;22:979-90.

17. Bamgbade OA, Adeogun BO, Abbas K. Fast-track laparoscopic gastric bypass surgery: outcomes and lessons from a bariatric surgery service in the United Kingdom. Obes Surg. 2012;22(3):398402.

18. Helmio M, Victorzon M, Ovaska J, et al. SLEEVEPASS: a randomized prospective multicenter study comparing laparoscopic sleeve gastrectomy and gastric bypass in the treatment of morbid obesity: preliminary results. Surg Endosc. 2012;26:2521-6.

19. Brooks-Brunn JA. Predictors of postoperative pulmonary complications following abdominal surgery. Chest. 1997;111:564-71.

20. Dimick JB, Chen SL, Taheri PA, et al. Hospital costs associated with surgical complications: a report from the private-sector National Surgical Quality Improvement Program. J Am Coll Surg. 2004;199:531-7.

21. Kehlet H, Wilmore DW. Multimodal strategies to improve surgical outcome. Am J Surg. 2002;183:630-41.

22. Lemanu DP, Singh PP, Berridge K, et al. Randomized clinical trial of enhanced recovery versus standard care after laparoscopic sleeve gastrectomy. Br J Surg. 2013;100:482-9.

23. Serafini F, Anderson W, Ghassemi P, et al. The utility of contrast studies and drains in the management of patients after Roux-en-Y gastric bypass. Obes Surg. 2002;12:34-8.

24. Shaffer D, Benotti PN, Bothe Jr A, et al. A prospective, randomized trial of abdominal wound drainage in gastric bypass surgery. Ann Surg. 1987;206:134-7.

25. Messager M, Sabbagh C, Denost $Q$, et al. Is there still a need for prophylactic intra-abdominal drainage in elective major gastrointestinal surgery? J Visc Surg. 2015;152:305-13.

26. Shin RB. Evaluation of the learning curve for laparoscopic Rouxen-Y gastric bypass surgery. Surg Obes Relat Dis. 2005;1:91-4.

27. Mosen DM, Schatz M, Magid DJ, Camargo CA,Jr. The relationship between obesity and asthma severity and control in adults. J Allergy Clin Immunol 2008; 122:507,11.e6.

28. van Huisstede A, Biter LU, Luitwieler R, et al. Pulmonary function testing and complications of laparoscopic bariatric surgery. Obes Surg. 2013;23:1596-603.

29. Greenburg DL, Lettieri CJ, Eliasson AH. Effects of surgical weight loss on measures of obstructive sleep apnea: a meta-analysis. Am J Med. 2009; 122:535-42.

30. Boulet LP, Turcotte H, Martin J, et al. Effect of bariatric surgery on airway response and lung function in obese subjects with asthma. Respir Med. 2012;106:651-60.

31. Kahokehr A, Hill AG. Early post-operative fever. ANZ J Surg. 2011;81:5-6. 